\title{
The tumour hypoxia marker pimonidazole reflects a transcriptional programme associated with aggressive prostate cancer
}

\author{
H B Ragnum ${ }^{1}$, L Vlatkovic ${ }^{2}$, A K Lie ${ }^{2}, \mathrm{~K} \mathrm{Axcrona}^{3}$, C H Julin ${ }^{1}, \mathrm{~K} \mathrm{M} \mathrm{Frikstad}^{1}$, K H Hole ${ }^{4}$, T Seierstad $^{4}$ \\ and H Lyng*,1
}

${ }^{1}$ Department of Radiation Biology, Norwegian Radium Hospital, Oslo University Hospital, Pb 4950, Nydalen, Oslo 0424, Norway; ${ }^{2}$ Department of Pathology, Norwegian Radium Hospital, Oslo University Hospital, Oslo, Norway; ${ }^{3}$ Department of Urology, Norwegian Radium Hospital, Oslo University Hospital, Oslo, Norway and ${ }^{4}$ Department of Radiology and Nuclear Medicine, Norwegian Radium Hospital, Oslo University Hospital, Oslo, Norway

Background: The hypoxia marker pimonidazole is a candidate biomarker of cancer aggressiveness. We investigated the transcriptional programme associated with pimonidazole staining in prostate cancer.

Methods: Index tumour biopsies were taken by image guidance from an investigation cohort of 52 patients, where 43 patients received pimonidazole before prostatectomy. Biopsy location within the index tumour was verified for 46 (88\%) patients, who were included for gene expression profiling and immunohistochemistry. Two independent cohorts of 59 and 281 patients were used for validation.

Results: Expression of genes in proliferation, DNA repair and hypoxia response was a major part of the transcriptional programme associated with pimonidazole staining. A signature of 32 essential genes was constructed and showed positive correlation to Ki67 staining, confirming the increased proliferation in hypoxic tumours as suggested from the gene data. Positive correlations were also found to tumour stage and lymph node status, but not to blood prostate-specific antigen level, consistent with the findings for pimonidazole staining. The association with aggressiveness was confirmed in validation cohorts, where the signature correlated with Gleason score and had independent prognostic impact, respectively.

Conclusions: Pimonidazole staining reflects an aggressive hypoxic phenotype of prostate cancer characterised by upregulation of proliferation, DNA repair and hypoxia response genes.

Tumour hypoxia is associated with aggressive disease and treatment resistance in prostate cancer (Milosevic et al, 2012; Turaka et al, 2012). Current clinicopathological markers are insufficient to identify patients at risk of treatment failure (Damber and Aus, 2008; Sartori and Chan, 2014), and a hypoxia biomarker would be of value for the clinical decision making. The exogenous hypoxia marker pimonidazole is a 2-nitroimidazole compound, which forms covalent bonds with cellular macromolecules at oxygen levels below 1.3\% (Gross et al, 1995) and visualises poorly oxygenated regions in histological sections from tumours
(Kizaka-Kondoh and Konse-Nagasawa, 2009). Only two studies have reported on the marker in prostate cancer (Carnell et al, 2006; Hoskin et al, 2007). Increased pimonidazole staining was found in tumours with high Gleason score (Carnell et al, 2006). Although pimonidazole seems not to be feasible for routine use owing to difficulties in assay standardisation, this encourages further exploration of the marker and methods derived from it.

Molecular biomarkers such as gene signatures have shown promising results in risk stratification of prostate cancer patients (Sartori and Chan, 2014), but the current knowledge of gene

*Correspondence: Dr H Lyng; E-mail: heidi.lyng@rr-research.no

Received 30 July 2014; revised 15 October 2014; accepted 4 November 2014;

published online 2 December 2014

(C) 2015 Cancer Research UK. All rights reserved 0007 - 0920/15

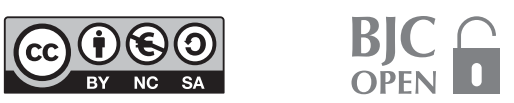


expression in hypoxic prostate tumours is scarce. By combined analysis of global expression profiles and pimonidazole data, a comprehensive picture of the transcriptional programme in pimonidazole-positive tumours can be achieved. This would increase our understanding of the hypoxic phenotype and would be of value in the development of a hypoxia biomarker. A major challenge in the use of biopsy-based biomarkers in prostate cancer is, however, the multifocality of the disease and pronounced intratumour heterogeneity in molecular abnormalities, which pose the need of sampling standardisation (Fraser et al, 2014). Recent advancements in image-guided technology facilitate sampling from the most aggressive sub-population within the tumours, that is, the index tumour (Sonn et al, 2013), which may solve this problem.

The present study was performed to explore the hypoxic phenotype associated with pimonidazole staining in prostate cancer. Based on pairwise gene expression and pimonidazole data, we determined the transcriptional programme of pimonidazolepositive tumours and constructed a signature with 32 essential genes. The investigation was based on a prostatectomy cohort, where correct prediction of index tumour by image guidance could be verified from the histopathology of the surgical specimen. We further explored the underlying biology of the signature and its ability to depict an aggressive tumour phenotype in independent cohorts.

\section{PATIENTS AND METHODS}

Investigation cohort. Fifty-two patients referred for robotassisted laparoscopic radical prostatectomy (RALP) from October 2011 to July 2012 at Oslo University Hospital, and recruited to our ongoing FuncProst-study (NCT01464216) (Supplementary Table S1) were used as investigation cohort to find pimonidazoleassociated genes. All patients had intermediate or high-risk disease according to the D'Amico classification (D’Amico et al, 1998). Patients who received hormonal therapy or radiotherapy before surgery were excluded. Written informed consent was obtained from all patients. The study was approved by the regional ethics committee and the protocol review committee of our institution.

The patients underwent preoperative, multiparametric magnetic resonance imaging (MRI) using a 1.5 T GE Discovery 450 (General Electric, Waukesha, WI, USA) magnet and phased array coils. The MRI protocol consisted of morphologic, T1- and T2-weighted (T1W/T2W), and functional, diffusion-weighted and dynamic contrast-enhanced (DW/DCE), sequences covering the pelvis and the lower abdomen. The protocol and image interpretation were in accordance with the European Society of Urogenital Radiology (ESUR) 2012 Guidelines (Barentsz et al, 2012). Lymph node status was determined based on node diameter and morphology in isotropic $1 \mathrm{~mm}$ T2W images.

Forty-three patients received $500 \mathrm{mg}$ pimonidazole hydrochloride (Hypoxyprobe Inc., Burlington, MA, USA) per $\mathrm{m}^{2}$ body surface in $100 \mathrm{ml} 0.9 \% \mathrm{NaCl}$ intravenously over $20 \mathrm{~min}$ the day before RALP. The maximum dose was $1000 \mathrm{mg}$, except for two patients who received 1025 and $1150 \mathrm{mg}$. The half-time of pimonidazole in blood is $5.1 \mathrm{~h}$ (http://www.hypoxyprobe.com). No patients experienced any side effects during or after the infusion. For the remaining nine patients in the cohort, pimonidazole infusion was not possible due to either anaphylactoid reaction to MR contrast agent or absence of study personnel, or correct quantification of pimonidazole staining was not possible due to postponing of surgery.

A three-armed robotic DaVinci system (Intuitive Surgical, Sunnyvale, CA, USA) was used to perform RALP (Axcrona et al, 2012). Pelvic lymph node dissection was generally performed in high-risk patients or when preoperative MRI had raised suspicion of lymph node metastasis. Lymph node status was thus determined either by pathological examination of lymph nodes or considered negative if preoperative MRI was negative and serum level of prostate-specific antigen (PSA) 6 weeks after prostatectomy was undetectable (Supplementary Table S1). Time from pimonidazole infusion to complete dissection of the prostate was recorded. The prostate was placed on ice immediately after RALP and transported to the pathology department.

Index tumour biopsies. Index tumour was predicted in MR images based on a combination of low signal intensity (SI) in T2W and T1W images, low apparent diffusion coefficient (ADC) and high SI in heavily DW images, and rapid wash-in and wash-out in DCE images (Figure 1A and B). By guidance from palpation of the prostate, preoperative biopsies and multiparametric MRI, the prostate was cut horizontally into two halves where the assumed index tumour was located. Two punch biopsies, $6 \mathrm{~mm}$ in diameter, were taken from the tumour, immediately snap frozen in liquid nitrogen and stored at $-80{ }^{\circ} \mathrm{C}$ (Figure 1C). The prostate specimens were fixed in $10 \%$ buffered formalin for at least $48 \mathrm{~h}$, and grossing was performed according to a standardised protocol (Srigley, 2006). Histopathological staging and grading were performed according to the TNM classification (Sobin et al, 2009) and a modified Gleason score system (Epstein, 2010). In case of multifocal tumour, index lesion was defined as, in descending order, pathologic $\mathrm{T}$ stage, Gleason score and tumour size, as discussed in van der Kwast et al (2011). By review of the pathology reports, one or both of the punch biopsies were verified to be from index tumour in $46(88 \%)$ of the patients, who were included in further analyses. Out of these, 39 patients had received pimonidazole. The biopsy with highest Gleason score within the index tumour was selected for gene expression and immunohistochemistry (Figure 1D), for which all had at least 75\% malignant glands and $<50 \%$ stroma in haematoxylin and eosin (HE)-stained sections (Figure 1E). Time from complete dissection of the prostate during RALP to snap-freezing of biopsies was recorded and denoted ischaemia time.

Immunohistochemistry. Immunohistochemistry was performed with monoclonal mouse antibodies for pimonidazole (1:50; Hypoxyprobe Inc.) on 39 tumours and Ki67 (1:75, MIB-1; Dako, Glostrup, Denmark) on 46 tumours using standard protocols extensively used at our institution. Pimonidazole antibody concentration was titrated to achieve high dynamic in staining intensity across the tumours. After thawing, sections were fixed in formalin for $15 \mathrm{~min}$ and rinsed in running water. Antigen retrieval was performed through boiling in citrate buffer $(\mathrm{pH}$ 6.0) in a microwave oven for $15 \mathrm{~min}$. Endogenous tissue peroxidase was quenched with hydrogen peroxide before incubation with antibodies for $30 \mathrm{~min}$ in room temperature. For visualisation, Envision system (Dako) was used, followed by counterstaining with haematoxylin, dehydration and mounting. One of the positive tumours was selected as a biological positive control and included in each run, ensuring satisfactory staining reproducibility. As a negative control, the primary antibodies were replaced with mouse myeloma proteins of equal subclasses and immunoglobulin concentrations. In addition, a patient who had received no pimonidazole served as a biological negative control for pimonidazole staining. None of the negative controls showed any reactivity.

The staining pattern of the malignant glands was evaluated blinded to gene expression and other immunohistochemistry data. Pimonidazole scoring was performed independently by two study pathologists (LV and AKL). Fractions of nuclear and moderate to strong cytoplasmic staining were determined separately and given immunoscores from 0 to 5 (0: $0 \% ; 1: 1-10 \% ; 2: 11-50 \%$; $3: 51-90 \%$; 4: $91-100 \%$; and 5: 100\%). It was a good agreement between the data of the two pathologists $(\kappa=0.80 ; P<0.0001$; Supplementary 


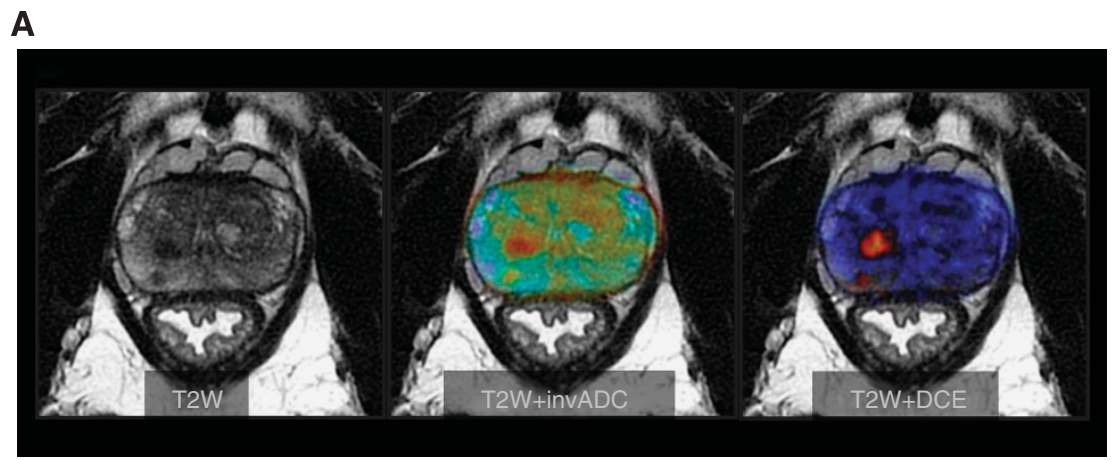

B
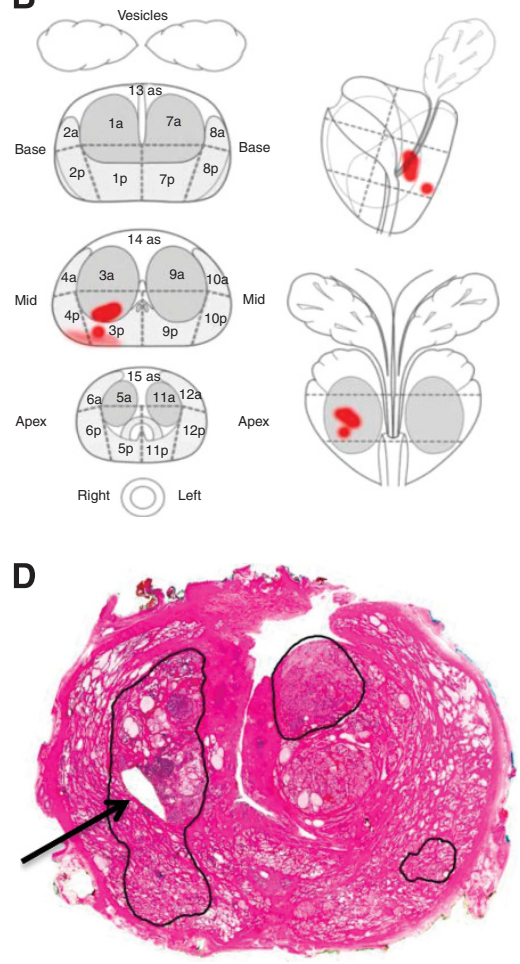

c

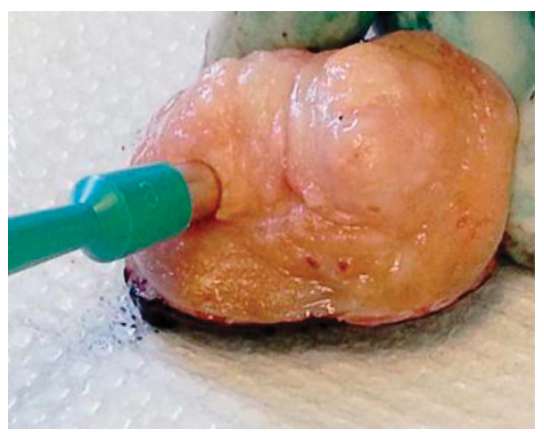

E

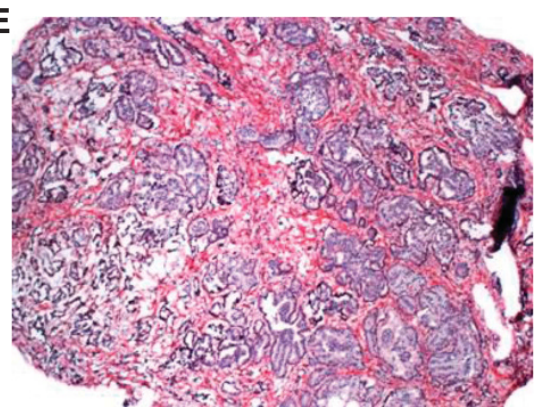

Figure 1. Biobanking of punch biopsies from index tumour. (A) Multiparametric MRI, left to right: T2W image, T2W image with inverted ADC-map overlaid and T2W image with DCE image overlaid. (B) Magnetic resonance imaging report based on the images in (A), indicating assumed index tumour foci (high - red) and more uncertain areas (pink). (C) Punch biopsy taken from assumed index tumour focus. (D) Whole-mount HE section with cancer foci (black circles) and punch biopsy hole (arrow) from index tumour, correctly estimated by MRI. (E) Haematoxylin and eosin section of a punch biopsy, demonstrating $>75 \%$ malignant glands and $<50 \%$ stroma (original magnification $\times 100$ ).

Figure S1), and in cases of disagreement, a consensus value was determined. The average value of cytoplasmic and nuclear immunoscore was used as the pimonidazole-positive fraction. Ki67 staining was scored by one of the pathologist (LV) in hotspots identified at low magnification. At least 500 tumour cells (median 610, range 501-963 cells) were evaluated in printed pictures at $\times 400$ magnification. Ki67 labelling index was calculated as the number of positive nuclei divided by the total number of nuclei.

Cell lines and hypoxia treatment. The human prostate cancer cell lines 22Rv1, LNCaP, PC-3 and DU 145 were used to generate prostate cancer-specific sets of hypoxia-responsive genes. The cell lines were cultured, routinely tested for mycoplasma and identity confirmed by short tandem repeat profiling as described by Ragnum et al (2013). Hypoxia treatment $\left(0.2 \% \mathrm{O}_{2}, 95 \% \mathrm{~N}_{2}, 5 \%\right.$ $\mathrm{CO}_{2}$ ) of exponentially growing cells at $60-70 \%$ cell confluence was carried out in an In Vivo 200 chamber (Ruskinn Technology, Brigend, UK) for $24 \mathrm{~h}$ at $37^{\circ} \mathrm{C}$. Cells were plated and incubated for
2 days, following medium replacement and hypoxia exposure. Normoxia samples (95\% air, 5\% $\mathrm{CO}_{2}$ ) were run in parallel.

Gene expression profiles. Gene expression profiles from freshfrozen biopsy sections of 46 tumours and normoxia- and hypoxiatreated cell lines were derived using Illumina bead arrays HT-12 v4 (Illumina Inc., San Diego, CA, USA) with 47300 transcripts, as described by Halle et al (2012). Total RNA was isolated using the RNeasy MiniKit (Qiagen, Hilden, Germany). RNA quality control was performed with Agilent 2100 Bioanalyzer (Agilent Technologies, Santa Clara, CA, USA). cRNA was synthesised, labelled and hybridised to the arrays. Signal extraction and quantile normalisation were carried out using the software provided by the manufacturer (Illumina Inc.). Log-transformed data were used in all analyses. The data have been deposited to the Gene Expression Omnibus (GEO) repository (GSE55935).

Computational analysis of gene expression. Gene expression profiles were combined with pimonidazole data in a two-step procedure described previously (Halle et al, 2012). First, an 
explorative, unsupervised analysis was performed. The investigation cohort was split into a pimonidazole-positive and -negative group according to an immunoscore of $<2(n=21)$ or $\geqslant 2$ $(n=18)$, and the Linear Models for Microarray Data software was applied to find genes differentially expressed between the groups. A nominal $P$-value of 0.05 was used as cutoff, resulting in an appropriate number of about 1000 genes. Biological processes enriched in the pimonidazole-positive group were analysed using the DAVID gene ontology (GO) software (Huang et al, 2009), where a false discovery rate of $<10 \%(q<0.1)$ was considered to be significant.

Second, a supervised gene set enrichment analysis was performed with 21 gene sets covering the significant biological processes from the GO analysis, using the Significance Analysis of Microarrays for Gene Sets (SAM-GS) software, which is based on the moderated t-statistics in SAM (Dinu et al, 2007). All gene sets were collected from the Molecular Signatures Database except a prostate cancer-specific hypoxia gene set constructed in this work, two hypoxia gene sets constructed in head and neck (Toustrup et al, 2011) and cervical cancer (Halle et al, 2012) and two target gene sets of the hypoxia-inducible factor 1 (HIF1) (Ragnum et al, 2013) and androgen receptor (AR) (Massie et al, 2011), respectively. The prostate cancer-specific hypoxia gene set was generated from the expression data of four prostate cancer cell lines and included genes with more than two-fold upregulation under hypoxia in at least two cell lines (Supplementary Table S2).

Validation cohorts. To validate results from the investigation cohort, two prostate cancer gene expression data sets in GEO with clinical information related to disease aggressiveness were applied (GSE32571 and GSE16560). In GSE32571, Illumina gene expression of about 48000 transcripts was available for 59 patients, who were grouped according to a Gleason score $\leqslant 7 \mathrm{a}$ or $\geqslant 7 \mathrm{~b}$ (Kuner et al, 2013). The Gleason score ranged from 5 to 10 , where the majority of the patients had a score of $7 \mathrm{a}(n=28)$ or $7 \mathrm{~b}$ and above $(n=27)$. GSE16560 contained data of 281 patients in a Swedish watchful waiting cohort, including expression of 6100 genes from the cDNA-mediated annealing, selection, ligation and extension (DASL) platform and up to 30 years follow-up for survival (Sboner et al, 2010). The patients had either died from prostate cancer or survived more than 10 years without metastases.

Statistics. Spearman's rank correlation and Mann-Whitney $U$-tests were applied on immunohistochemistry data, and Student's $t$-test was used on continuous data. Associations between categorical data were analysed by $\chi^{2}$ test. Kaplan-Meier curves were compared with log-rank test. Multivariate regression analysis was performed using Cox proportional hazards model. Cohen's kappa $(\kappa)$ was used to evaluate the agreement between immunoscores obtained from two different pathologists. PASW Statistics 18 for Windows (SPSS Inc., Chicago, IL, USA) was used with a significance level of 0.05 , unless otherwise specified.

\section{RESULTS}

Pimonidazole staining in relation to tumour aggressiveness and gene expression. The pimonidazole staining pattern differed considerably among tumours (Figure $2 \mathrm{~A}$ and $\mathrm{B}$ ). Median immunoscore was 2 , and 18 cases (46\%) had an immunoscore of 2 or higher, implying a pimonidazole-positive fraction above $10 \%$. The immunoscore was significantly higher for tumours at a high clinical stage $(P=0.019$; Figure $2 \mathrm{C})$ and with lymph node metastasis $(P=0.033$; Figure $2 \mathrm{D})$, suggesting an association between pimonidazole staining and tumour aggressiveness. Staining was not correlated with time from pimonidazole infusion to complete dissection of the prostate (Supplementary Figure S2A), blood PSA level or ischaemia time (data not shown).

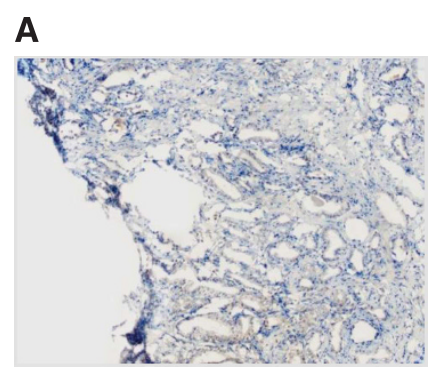

B
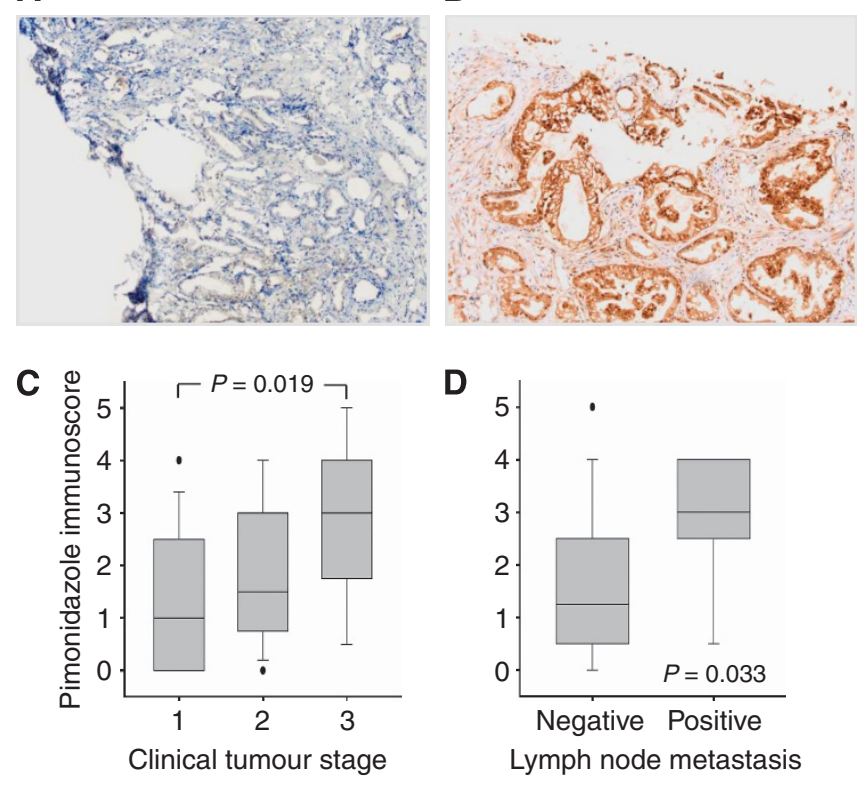

Figure 2. Pimonidazole staining in relation to clinicopathological parameters. (A and B) Histological section of index tumour biopsy from two different patients, showing low and high pimonidazole immunoscore of 0 and 5, respectively (original magnifications $\times 100$ ). (C and D) Box plot of pimonidazole immunoscore at different clinical tumour stage (left; cT1, $n=20 ; c T 2, n=14 ; c T 3, n=10$ ) and lymph node status (right; negative, $n=36$; positive, $n=8$ ). Data were available for 44 patients. $P$-values from Mann-Whitney U-tests are displayed. In (C), $P$-value refers to $\mathrm{CT} 3$ vs cT1.

To obtain a general picture of the biology of pimonidazolepositive tumours, all differentially expressed genes between pimonidazole-positive and -negative tumours were included in an unsupervised GO analysis. Three major, significant biological processes were identified based on the 1046 genes that were upregulated in pimonidazole-positive tumours: cell cycle, translation and cellular response to stress, including the subgroups DNA repair and response to DNA damage stimulus (Supplementary Table S3). A similar analysis of 862 downregulated genes identified only the process muscle system, whereas analysis of up- and downregulated genes combined showed no significant processes.

To address specifically all likely phenotypes of the pimonidazole-positive tumours, we used a supervised gene set analysis based on all genes on the array and 21 gene sets representing the significant biological processes from the GO analysis. Our prostate cancer-specific hypoxia gene set (Supplementary Table S2), six published hypoxia gene sets (Mense et al, 2006; Winter et al, 2007; Buffa et al, 2010; Toustrup et al, 2011; Halle et al, 2012; Eustace et al, 2013) and two sets of HIF1 and AR targets (Massie et al, 2011; Ragnum et al, 2013) were included to obtain a wide coverage of the hypoxia phenotype. To cover the cell cycle process, the reactome cell cycle checkpoint pathway and two published proliferation gene sets (Chiang et al, 2008; Starmans et al, 2008) were applied. We further used the Kyoto Encyclopedia of Genes and Genomes (KEGG) pathway for protein processing in endoplasmic reticulum to represent translation and eight sets of genes in various DNA repair pathways from the KEGG and GO databases to cover cellular response to stress and its subgroups.

The two proliferation gene sets showed the strongest association with pimonidazole immunoscore, followed by two DNA repair gene sets and the prostate-specific hypoxia gene set (Table 1). These five gene sets covered all significant biological processes from the GO analysis (Supplementary Table S3). Expression of genes in proliferation, repair and hypoxia response therefore 


\begin{tabular}{|c|c|c|c|}
\hline Gene set ${ }^{a}$ & Phenotype & No. of genes & $P$-value \\
\hline STARMANS_PROLIFERATION_SIGNATURE & Proliferation & 104 & 0.013 \\
\hline CHIANG_LIVER_CANCER_SUBCLASS_PROLIFERATION_UP & Proliferation & 177 & 0.014 \\
\hline GO_BASE_EXCISION_REPAIR & DNA repair & 17 & 0.082 \\
\hline KEGG_NON_HOMOLOGOUS_END_JOINING & DNA repair & 13 & 0.088 \\
\hline HYPOXIA_UP_PROSTATE_CANCER_IN_VITRO & Hypoxia & 107 & 0.090 \\
\hline BUFFA_HYPOXIA_METAGENE & Hypoxia & 51 & 0.10 \\
\hline REACTOME_CELL_CYCLE_CHECKPOINTS & Proliferation & 110 & 0.11 \\
\hline MENSE_HYPOXIA_UP & Hypoxia & 95 & 0.11 \\
\hline KEGG_HOMOLOGOUS_RECOMBINATION & DNA repair & 27 & 0.11 \\
\hline KEGG_MISMATCH_REPAIR & DNA repair & 23 & 0.16 \\
\hline KEGG_BASE_EXCISION_REPAIR & DNA repair & 32 & 0.17 \\
\hline HIF1_TARGETS & HIF1 pathway & 273 & 0.20 \\
\hline GO_DOUBLE_STRAND_BREAK_REPAIR & DNA repair & 23 & 0.21 \\
\hline TOUSTRUP_HYPOXIA_IN_VIVO & Hypoxia & 15 & 0.22 \\
\hline AR_TARGETS & AR pathway & 1115 & 0.24 \\
\hline EUSTACE_HYPOXIA_CLASSIFIER & Hypoxia & 25 & 0.27 \\
\hline HALLE_HYPOXIA_IN_VIVO & Hypoxia & 31 & 0.30 \\
\hline KEGG_NUCLEOTIDE_EXCISION_REPAIR & DNA repair & 44 & 0.33 \\
\hline KEGG_PROTEIN_PROCESSING_IN_ER & Translation & 163 & 0.33 \\
\hline WINTER_HYPOXIA_UP & Hypoxia & 91 & 0.42 \\
\hline GO_NUCLEOTIDE_EXCISION_REPAIR & DNA repair & 20 & 0.59 \\
\hline
\end{tabular}

seemed to pose a major contribution to the transcriptional programme reflected by pimonidazole staining.

Construction of a pimonidazole gene signature. To find essential genes reflected by pimonidazole staining, we selected the five most significant gene sets covering the three phenotypes proliferation, repair and hypoxia response (Table 1), and extracted the 32 genes with a positive correlation to pimonidazole immunoscore $(P<0.05$; Table 2$)$. Negatively correlated genes were few (four in proliferation, one in repair, two in hypoxia response) and not included owing to the lack of significance for downregulated genes in the GO analysis. Several of the signature genes were direct HIF1 or AR targets (Massie et al, 2011; Halle et al, 2012; Xia et al, 2012; Gilkes et al, 2013; Ragnum et al, 2013), suggesting that both the HIF1 and AR pathways were important parts of the transcriptional programme and activated in pimonidazole-positive tumours.

To confirm that the gene signature sufficiently reflected pimonidazole staining, unsupervised hierarchical clustering of the investigation cohort based on the 32 signature genes was performed. This produced two clusters, for which the cluster with high expression had higher frequency of pimonidazolepositive tumours $(P=0.017$; Figure $3 \mathrm{~A})$ and increased immunoscore $(P=0.002$; data not shown). We further calculated a pimonidazole gene score for each tumour by averaging the median-centred, log-transformed expression levels of the genes, to achieve a measure of the signature that could be compared in other cohorts. The gene score was higher for pimonidazole-positive tumours $(P<0.001$; data not shown $)$ and in patients with high clinical stage $(P=0.031$ and $P=0.002$ for cT3 vs cT2 and cT1, respectively; Figure $3 \mathrm{~B})$ and lymph node metastasis $(P=0.001$; Figure $3 \mathrm{C})$. A correlation between gene score and Gleason score in the surgical specimens was also observed $(P=0.033$; data not shown). There was no relationship between the signature and ischaemia time (Supplementary Figures S2B and C), time from pimonidazole infusion to complete dissection of the prostate or blood PSA level (data not shown). The gene signature therefore seemed to satisfactorily represent pimonidazole staining, including its relationship to aggressiveness in the investigation cohort.

Pimonidazole gene signature in relation to Ki67 labelling index. The contribution of proliferation genes to the transcriptional programme of pimonidazole-positive tumours (Table 1) suggested increased proliferation activity. To validate this, the pimonidazole gene signature was analysed against the Ki67 proliferation marker. A significant positive correlation $(P<0.05)$ between gene expression and Ki67 labelling index was found for 14 signature genes, including eight proliferation genes, all three DNA repair genes and three hypoxia response genes, whereas no genes showed a significant negative correlation (Table 2). A significant correlation was also found for the gene score $(P=0.001$; Figure 4A). Moreover, Ki67 staining was often observed in pimonidazole-positive regions within tumours (Figure 4B). The increased proliferation associated with pimonidazole staining, as suggested from the gene data, was therefore confirmed. However, relatively high Ki67 labelling index was also seen in some tumours with low gene score (Figure 4A), and the difference in labelling index between pimonidazole-positive and -negative tumours was not significant but only a tendency was observed $(P=0.17$; data not shown). These observations support the contribution of other features covered by the gene signature, that is, DNA repair and hypoxia response, in addition to increased proliferation.

Validation of the pimonidazole gene signature in independent cohorts. To validate the relationship between the pimonidazole gene signature and aggressiveness, we first investigated its association with Gleason score in the GSE32571 cohort, where all 32 signature genes were available. A significantly higher gene 
Table 2. Pimonidazole gene signature

\begin{tabular}{|c|c|c|c|c|c|c|}
\hline \multirow[b]{2}{*}{ ProbelD ${ }^{a}$} & \multirow[b]{2}{*}{ Gene symbol ${ }^{b}$} & \multirow[b]{2}{*}{ Gene name } & \multicolumn{2}{|c|}{ Pimonidazole } & \multicolumn{2}{|c|}{ Ki67 } \\
\hline & & & Corr $^{c}$ & $P$-value ${ }^{\mathrm{c}}$ & Corr $^{c}$ & $P$-value ${ }^{c}$ \\
\hline \multicolumn{7}{|c|}{ Proliferation gene sets ${ }^{d}$} \\
\hline 2630673 & ASF1B & Antisilencing function $1 \mathrm{~B}$ histone chaperone & 0.39 & 0.013 & 0.29 & 0.052 \\
\hline 6130441 & ASPM & Abnormal spindle homolog, microcephaly associated (Drosophila) & 0.39 & 0.016 & 0.57 & $<0.001$ \\
\hline 1230682 & BIRC5 & Baculoviral IAP repeat containing 5 & 0.36 & 0.023 & 0.33 & 0.026 \\
\hline 6350348 & BUB3 & BUB3 mitotic checkpoint protein & 0.35 & 0.029 & 0.29 & 0.048 \\
\hline 2510639 & CENPE & Centromere protein E, $312 \mathrm{kDa}$ & 0.45 & 0.004 & 0.26 & 0.078 \\
\hline 2120678 & CENPU & Centromere protein $U$ & 0.33 & 0.043 & 0.17 & 0.27 \\
\hline 6020746 & СMTM3 & CKLF-like MARVEL transmembrane domain containing 3 & 0.49 & 0.002 & 0.20 & 0.18 \\
\hline 990725 & DONSON & Downstream neighbor of SON & 0.33 & 0.039 & 0.41 & 0.005 \\
\hline 5570296 & DTL & Denticleless E3 ubiquitin protein ligase homolog (Drosophila) & 0.42 & 0.008 & -0.07 & 0.65 \\
\hline 540053 & FOXM1 & Forkhead box M1 & 0.39 & 0.014 & 0.31 & 0.034 \\
\hline 6520095 & G6PD & Glucose-6-phosphate dehydrogenase & 0.38 & 0.018 & -0.10 & 0.49 \\
\hline 3180367 & HJURP & Holliday junction recognition protein & 0.38 & 0.018 & 0.54 & $<0.001$ \\
\hline 6770408 & MCM2 & Minichromosome maintenance complex component 2 & 0.52 & 0.001 & 0.42 & 0.003 \\
\hline 1820176 & MEP1A & Meprin A, alpha (PABA peptide hydrolase) & 0.32 & 0.047 & 0.28 & 0.058 \\
\hline 3190768 & MTMR2 & Myotubularin-related protein 2 & 0.32 & 0.049 & -0.12 & 0.42 \\
\hline 5700373 & $\overline{\text { TRIP13 }}$ & Thyroid hormone receptor interactor 13 & 0.34 & 0.037 & 0.54 & $<0.001$ \\
\hline 650156 & ZWINT & ZW10-interacting kinetochore protein & 0.33 & 0.042 & -0.03 & 0.85 \\
\hline \multicolumn{7}{|c|}{ DNA repair gene sets ${ }^{d}$} \\
\hline 1990253 & TDG & Thymine-DNA glycosylase & 0.39 & 0.014 & 0.38 & 0.009 \\
\hline 3610750 & UNG & Uracil-DNA glycosylase & 0.36 & 0.024 & 0.32 & 0.033 \\
\hline 430100 & $\overline{\mathrm{XRCC} 6}$ & $X$-ray repair complementing defective repair in Chinese hamster cells 6 & 0.40 & 0.011 & 0.43 & 0.003 \\
\hline \multicolumn{7}{|c|}{ Hypoxia gene set ${ }^{d}$} \\
\hline 5670465 & ADM & Adrenomedullin & 0.36 & 0.023 & 0.06 & 0.69 \\
\hline 3190148 & $\overline{\mathrm{DDIT}} 4$ & DNA-damage-inducible transcript 4 & 0.44 & 0.005 & 0.34 & 0.02 \\
\hline 3610672 & DSP & Desmoplakin & 0.35 & 0.03 & 0.23 & 0.13 \\
\hline 4640039 & FER1L4 & Fer-1-like 4 (C. elegans), pseudogene & 0.42 & 0.008 & 0.05 & 0.72 \\
\hline 7320441 & HILPDA & Hypoxia-inducible lipid droplet-associated & 0.37 & 0.021 & 0.03 & 0.87 \\
\hline 4220731 & P4HA1 & Prolyl 4-hydroxylase, alpha polypeptide I & 0.38 & 0.018 & 0.35 & 0.017 \\
\hline 1820424 & PGAM4 & Phosphoglycerate mutase family member 4 & 0.36 & 0.024 & 0.05 & 0.73 \\
\hline 2030093 & $\mathrm{PKM}^{\mathrm{e}}$ & Pyruvate kinase, muscle & 0.42 & 0.009 & 0.22 & 0.14 \\
\hline 6840619 & RIMKLA & Ribosomal modification protein rimK-like family member $A$ & 0.39 & 0.014 & 0.40 & 0.006 \\
\hline 3930372 & RNASE4 & Ribonuclease, RNase A family, 4 & 0.46 & 0.004 & 0.10 & 0.50 \\
\hline 2140128 & SCD & Stearoyl-CoA desaturase (delta-9-desaturase) & 0.37 & 0.022 & 0.04 & 0.77 \\
\hline 1190670 & SPAG4 & Sperm-associated antigen 4 & 0.32 & 0.047 & 0.14 & 0.37 \\
\hline \multicolumn{7}{|c|}{ 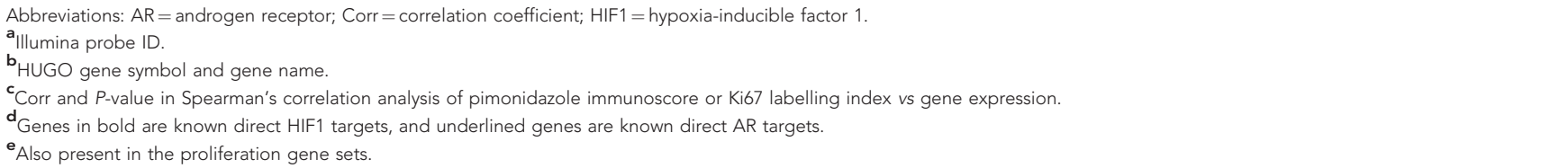 } \\
\hline
\end{tabular}

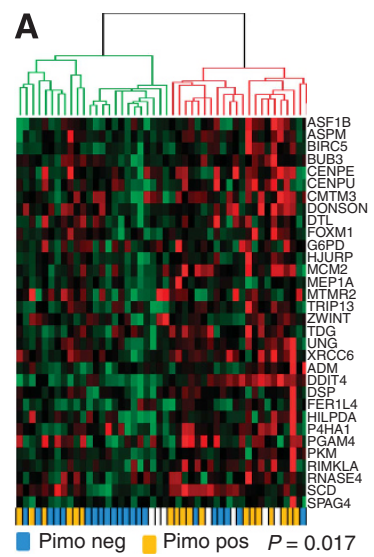

B

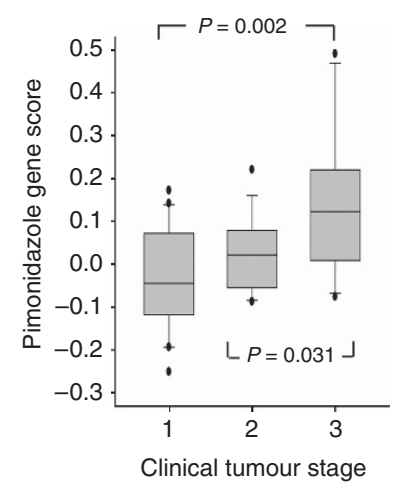

C

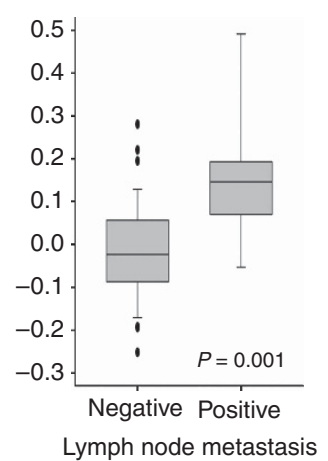

Figure 3. Pimonidazole gene signature in relation to pimonidazole staining and tumour aggressiveness. (A) Hierarchical clustering of patients (columns) based on the pimonidazole gene signature (rows). Red, green: high, low expression relative to median expression level, respectively; blue, yellow: pimonidazole-negative, -positive tumour, respectively; white: tumours without pimonidazole data. (B and C) Box plot of pimonidazole gene score at different clinical tumour stages (cT1, $n=20 ; c T 2, n=14 ; c T 3, n=10$ ) and lymph node status (negative, $n=36 ;$ positive, $n=8$ ). Data were available for 44 patients. $P$-values from $\chi^{2}$-test $(\mathbf{A})$ and $t$-tests $(\mathbf{B}$ and $\mathbf{C})$ are displayed. 
score was seen in the high Gleason group, as compared with the low one $(P=0.002$; Figure $5 \mathrm{~A})$. In the second validation cohort (GSE16560), only 19 signature genes were present, and we first confirmed that the gene score based on this subgroup of genes sufficiently reflected pimonidazole staining in the investigation cohort (Supplementary Figure S3). Thereafter, a significant relationship between the gene score and outcome was
A

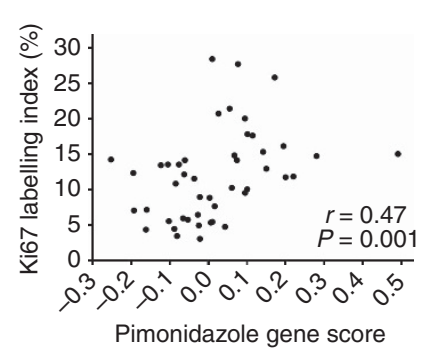

B

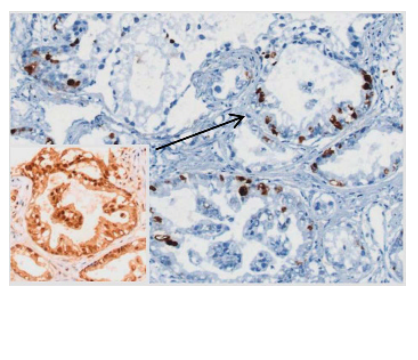

Figure 4. Pimonidazole gene signature in relation to Ki67 staining. (A) Ki67 labelling index vs pimonidazole gene score. Correlation coefficient $(r)$ and $P$-value from Spearman's correlation analysis are shown. (B) Histological section showing Ki67 staining of the pimonidazole-positive tumour in Figure 2B with a Ki67 labelling index of $16.1 \%$ and gene score of 0.19 (original magnification $\times 100$ ). Inset, pimonidazole staining of Ki67-positive malignant gland. The arrow points to the positive gland.
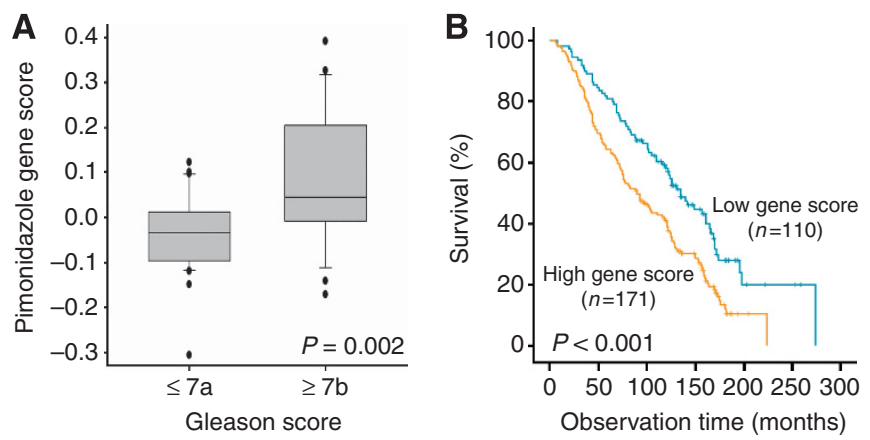

Figure 5. Validation of the pimonidazole gene signature in two independent prostate cancer cohorts. (A) Box plot of pimonidazole gene score for patients in the GSE32571 cohort with a Gleason score $\leqslant 7 \mathrm{a}(n=32)$ and $\geqslant 7 \mathrm{~b}(n=27)$. P-value from $t$-test is displayed. (B) Kaplan-Meier curves for survival of 281 patients in the GSE16560 cohort. Number of patients and P-values from log-rank tests are displayed. demonstrated in the GSE16560 cohort, both in log-rank test $(P<0.001$; Figure 5B) and as a continuous variable in Cox univariate analysis $(P<0.001$; Table 3$)$. The relationships to aggressiveness were also seen for both validation cohorts when the patients were clustered based on the signature genes (data not shown). The two validation experiments therefore confirmed a relationship between the pimonidazole gene signature and tumour aggressiveness.

To assess the importance of the pimonidazole gene signature corrected for available clinicopathological markers, a multivariate Cox analysis was carried out on the GSE16560 cohort. The signature showed prognostic significance independent of the other markers $(P<0.004$; Table 3$)$.

\section{DISCUSSION}

We have performed an integrative analysis of global gene expression and pimonidazole staining in prostate cancer. Pimonidazole staining visualises cumulative hypoxia over the time period from infusion to snap-freezing of biopsies, provided that pimonidazole is still available in the tissue for binding. Systematic recording of time from infusion to surgery and from surgery to snap-freezing of biopsies showed that these parameters had no significant influence on our results, most probably because they differed little across the patients or because only minor pimonidazole was available in the tumour at the time of surgery. The staining differences therefore seemed to reliably represent differences in pretreatment hypoxia. The staining pattern reflected a transcriptional programme activated by proliferation, DNA repair and hypoxia and included a gene signature associated with aggressive disease in two independent patient cohorts, pointing to signalling pathways that may promote tumour progression. We demonstrated that index tumour could be successfully identified by guidance from multiparametric MRI, enabling standardised sampling of pretreatment biopsies to assess the signature. Our work provides novel insight into the molecular background of the hypoxic phenotype reflected by pimonidazole staining, encouraging studies to clarify the potential of the gene signature in the decision making of patients with prostate cancer.

Gene expression associated with increased proliferation posed a major part of the transcriptional programme reflected by pimonidazole staining. This conclusion was based on results from both GO and gene set analyses, where the cell cycle process and proliferation gene sets were the most significant ones, respectively, and by the observed correlation between gene expression and Ki67 labelling index. Also, the upregulation of DNA repair genes could reflect increased proliferation, being a consequence of elevated replication stress (Ciccia and Elledge, 2010). In accordance

Table 3. Cox regression analysis of validation cohort ${ }^{a}$

\begin{tabular}{|c|c|c|c|c|c|c|}
\hline \multirow[b]{2}{*}{ Factor } & \multicolumn{3}{|c|}{ Univariate analysis } & \multicolumn{3}{|c|}{ Multivariate analysis } \\
\hline & $P$-value & Relative risk & $95 \% \mathrm{Cl}$ & $P$-value & Relative risk & $95 \% \mathrm{Cl}$ \\
\hline Gleason score $^{\mathbf{b}}$ & $<0.001$ & 1.84 & $1.63-2.09$ & $<0.001$ & 1.62 & $1.41-1.87$ \\
\hline Cancer percentage $^{\mathbf{b}}$ & $<0.001$ & 1.02 & $1.01-1.03$ & 0.041 & 1.01 & $1.00-1.01$ \\
\hline Age $^{b}$ & $<0.001$ & 1.06 & $1.04-1.08$ & $<0.001$ & 1.05 & $1.03-1.07$ \\
\hline TMPRSS-ERG status $^{c}$ & $<0.001$ & 2.32 & $1.65-3.28$ & $(0.06)$ & 1.44 & $0.99-2.10$ \\
\hline Pimonidazole gene score ${ }^{\mathbf{b}}$ & $<0.001$ & 6.23 & $3.00-12.93$ & 0.004 & 3.17 & $1.45-6.93$ \\
\hline \multicolumn{7}{|c|}{ 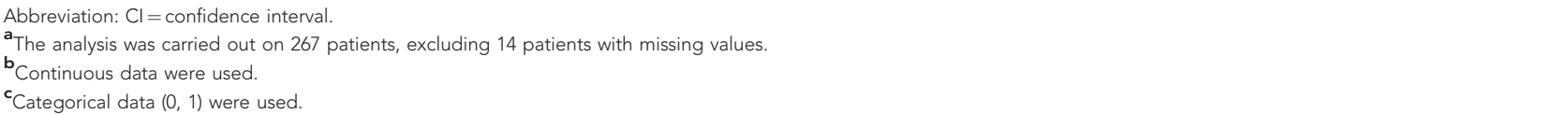 } \\
\hline
\end{tabular}


with our results, expression of the hypoxia-inducible lactate dehydrogenase 5 isoenzyme has been found to be elevated in the most proliferative prostate tumours (Koukourakis et al, 2014), supporting a positive relationship between proliferation and hypoxia in this disease.

The mechanisms underlying high proliferation capacity of pimonidazole-positive tumours is not clear. Upregulation of HIF1 and AR targets was part of the transcriptional programme reflected by pimonidazole staining. Similar to HIF1, AR is activated under hypoxia and promotes cell survival, metabolism and proliferation (Mitani et al, 2011; Park et al, 2012; Lee et al, 2013). Although severe hypoxia may induce cell cycle arrest and apoptosis, the oxygen levels reflected by pimonidazole staining seemed to be sufficiently high to allow proliferation, maintaining metabolic activity and oxygen consumption. A similar conclusion was drawn in a pimonidazole study on colorectal cancer metastases (van Laarhoven et al, 2006), but seemed not to be valid for head and neck cancer (Wijffels et al, 2008), indicating that this finding is tumour type-dependent. Alternatively, the high proliferation capacity and overlap between pimonidazole and Ki67 staining may be due to reoxygenation of hypoxic regions after irreversible pimonidazole binding.

Extraction of essential genes in the transcriptional programme resulted in a signature associated with aggressive features, including poor clinical outcome. The signature seemed to capture both elevated proliferation and hypoxia, which are two known aggressive prostate cancer phenotypes (Milosevic et al, 2012; Turaka et al, 2012; Verhoven et al, 2013). A gene set reflecting proliferation and hypoxia-related tissue remodelling was recently found to have strong association with survival of prostate cancer patients, as compared with a proliferation or hypoxia-related gene set alone (Markert et al, 2012). Pimonidazole staining possibly reflects oxygen levels that promote such an aggressive phenotype by covering both proliferation and hypoxia response. Moreover, our signature might be refined to become a marker of this aggressive hypoxic phenotype.

Little is known about how the individual signature genes influence prostate cancer progression. Upregulation of some genes or their encoded protein has been associated with aggressive disease (BIRC5, MCM2 and TRIP13) (Shariat et al, 2004; Larkin et al, 2012; Toubaji et al, 2012). Moreover, SCD has been shown to enhance AR transcriptional activity and thereby promote proliferation of prostate cancer cells (Kim et al, 2011), FOXM1 seems to have a role in prostate carcinogenesis (Chandran et al, 2007; Cai et al, 2013) and transcriptional upregulation of the AR target ZWINT has been associated with castration-resistant prostate cancer (Urbanucci et al, 2012). Altogether, these studies support the involvement of the genes in an aggressive prostate tumour phenotype.

In conclusion, pimonidazole-positive prostate cancer shows aggressive features, including activation of a transcriptional programme that promotes proliferation. The essential genes in this programme provide information on disease progression that is not correlated with the blood PSA level or covered by conventional clinical parameters such as Gleason score and by the TMPRSS-ERG fusion gene status. The gene signature might therefore be developed into a useful biomarker for identifying patients in need for intensified treatment, which possibly could include hypoxia targeting.

\section{ACKNOWLEDGEMENTS}

Technical assistance from Aa Nyboen, J-I Krog, M Nguyen and D Trinh is highly appreciated. Financial support was provided by the European Union 7th Framework Program (Grant No.
222741-METOXIA) and the Norwegian Cancer Society (Grant No. 107438-PR-20070179).

\section{CONFLICT OF INTEREST}

The authors declare no conflict of interest.

\section{REFERENCES}

Axcrona K, Vlatkovic L, Hovland J, Brennhovd B, Kongsgaard U, Giercksky KE (2012) Robot-assisted laparoscopic prostatectomy in a 68-year-old patient with previous heart transplantation and pelvic irradiation. J Robot Surg 6: 81-83.

Barentsz JO, Richenberg J, Clements R, Choyke P, Verma S, Villeirs G, Rouviere O, Logager V, Futterer JJ (2012) ESUR prostate MR guidelines 2012. Eur Radiol 22: 746-757.

Buffa FM, Harris AL, West CM, Miller CJ (2010) Large meta-analysis of multiple cancers reveals a common, compact and highly prognostic hypoxia metagene. Br J Cancer 102: 428-435.

Cai Y, Balli D, Ustiyan V, Fulford L, Hiller A, Misetic V, Zhang Y, Paluch AM, Waltz SE, Kasper S, Kalin TV (2013) Foxm1 expression in prostate epithelial cells is essential for prostate carcinogenesis. J Biol Chem 288: 22527-22541.

Carnell DM, Smith RE, Daley FM, Saunders MI, Bentzen SM, Hoskin PJ (2006) An immunohistochemical assessment of hypoxia in prostate carcinoma using pimonidazole: implications for radioresistance. Int J Radiat Oncol Biol Phys 65: 91-99.

Chandran UR, Ma C, Dhir R, Bisceglia M, Lyons-Weiler M, Liang W, Michalopoulos G, Becich M, Monzon FA (2007) Gene expression profiles of prostate cancer reveal involvement of multiple molecular pathways in the metastatic process. BMC Cancer 7: 64 .

Chiang DY, Villanueva A, Hoshida Y, Peix J, Newell P, Minguez B, LeBlanc AC, Donovan DJ, Thung SN, Sole M, Tovar V, Alsinet C, Ramos AH, Barretina J, Roayaie S, Schwartz M, Waxman S, Bruix J, Mazzaferro V, Ligon AH, Najfeld V, Friedman SL, Sellers WR, Meyerson M, Llovet JM (2008) Focal gains of VEGFA and molecular classification of hepatocellular carcinoma. Cancer Res 68: 6779-6788.

Ciccia A, Elledge SJ (2010) The DNA damage response: making it safe to play with knives. Mol Cell 40: 179-204.

D’Amico AV, Whittington R, Malkowicz SB, Schultz D, Blank K, Broderick GA, Tomaszewski JE, Renshaw AA, Kaplan I, Beard CJ, Wein A (1998) Biochemical outcome after radical prostatectomy, external beam radiation therapy, or interstitial radiation therapy for clinically localized prostate cancer. JAMA 280: 969-974.

Damber JE, Aus G (2008) Prostate cancer. Lancet 371: 1710-1721.

Dinu I, Potter JD, Mueller T, Liu Q, Adewale AJ, Jhangri GS, Einecke G, Famulski KS, Halloran P, Yasui Y (2007) Improving gene set analysis of microarray data by SAM-GS. BMC Bioinform 8: 242 .

Epstein JI (2010) An update of the gleason grading system. J Urol 183: $433-440$.

Eustace A, Mani N, Span PN, Irlam JJ, Taylor J, Betts GN, Denley H, Miller CJ, Homer JJ, Rojas AM, Hoskin PJ, Buffa FM, Harris AL, Kaanders JH, West CM (2013) A 26-gene hypoxia signature predicts benefit from hypoxia-modifying therapy in laryngeal cancer but not bladder cancer. Clin Cancer Res 19: 4879-4888.

Fraser M, Berlin A, Bristow RG, van der Kwast T (2014) Genomic, pathological, and clinical heterogeneity as drivers of personalized medicine in prostate cancer. Urol Oncol 32: S1078-S1439.

Gilkes DM, Bajpai S, Chaturvedi P, Wirtz D, Semenza GL (2013) Hypoxia-inducible factor 1 (HIF-1) promotes extracellular matrix remodeling under hypoxic conditions by inducing P4HA1, P4HA2, and PLOD2 expression in fibroblasts. J Biol Chem 288: 10819-10829.

Gross MW, Karbach U, Groebe K, Franko AJ, Mueller-Klieser W (1995) Calibration of misonidazole labeling by simultaneous measurement of oxygen tension and labeling density in multicellular spheroids. Int J Cancer 61: 567-573.

Halle C, Andersen E, Lando M, Aarnes EK, Hasvold G, Holden M, Syljuasen RG, Sundfor K, Kristensen GB, Holm R, Malinen E, Lyng H (2012) Hypoxiainduced gene expression in chemoradioresistant cervical cancer revealed by dynamic contrast-enhanced MRI. Cancer Res 72: 5285-5295. 
Hoskin PJ, Carnell DM, Taylor NJ, Smith RE, Stirling JJ, Daley FM, Saunders MI, Bentzen SM, Collins DJ, D'Arcy JA, Padhani AP (2007) Hypoxia in prostate cancer: correlation of BOLD-MRI with pimonidazole immunohistochemistry-initial observations. Int J Radiat Oncol Biol Phys 68: $1065-1071$.

Huang dW, Sherman BT, Lempicki RA (2009) Systematic and integrative analysis of large gene lists using DAVID bioinformatics resources. Nat Protoc 4: 44-57.

Kim SJ, Choi H, Park SS, Chang C, Kim E (2011) Stearoyl CoA desaturase (SCD) facilitates proliferation of prostate cancer cells through enhancement of androgen receptor transactivation. Mol Cells 31: 371-377.

Kizaka-Kondoh S, Konse-Nagasawa H (2009) Significance of nitroimidazole compounds and hypoxia-inducible factor-1 for imaging tumor hypoxia. Cancer Sci 100: 1366-1373.

Koukourakis MI, Giatromanolaki A, Panteliadou M, Pouliliou SE, Chondrou PS, Mavropoulou S, Sivridis E (2014) Lactate dehydrogenase 5 isoenzyme overexpression defines resistance of prostate cancer to radiotherapy. Br J Cancer 110: 2217-2223.

Kuner R, Falth M, Pressinotti NC, Brase JC, Puig SB, Metzger J, Gade S, Schafer G, Bartsch G, Steiner E, Klocker H, Sultmann H (2013) The maternal embryonic leucine zipper kinase (MELK) is upregulated in highgrade prostate cancer. J Mol Med (Berl) 91: 237-248.

Larkin SE, Holmes S, Cree IA, Walker T, Basketter V, Bickers B, Harris S, Garbis SD, Townsend PA, ukim-Hastie C (2012) Identification of markers of prostate cancer progression using candidate gene expression. Br J Cancer 106: 157-165.

Lee HY, Yang EG, Park H (2013) Hypoxia enhances the expression of prostate-specific antigen by modifying the quantity and catalytic activity of Jumonji C domain-containing histone demethylases. Carcinogenesis 34: 2706-2715.

Markert EK, Levine AJ, Vazquez A (2012) Proliferation and tissue remodeling in cancer: the hallmarks revisited. Cell Death Dis 3: e397.

Massie CE, Lynch A, Ramos-Montoya A, Boren J, Stark R, Fazli L, Warren A, Scott H, Madhu B, Sharma N, Bon H, Zecchini V, Smith DM, Denicola GM, Mathews N, Osborne M, Hadfield J, Macarthur S, Adryan B, Lyons SK, Brindle KM, Griffiths J, Gleave ME, Rennie PS, Neal DE, Mills IG (2011) The androgen receptor fuels prostate cancer by regulating central metabolism and biosynthesis. EMBO J 30: 2719-2733.

Mense SM, Sengupta A, Zhou M, Lan C, Bentsman G, Volsky DJ, Zhang L (2006) Gene expression profiling reveals the profound upregulation of hypoxia-responsive genes in primary human astrocytes. Physiol Genom 25: 435-449.

Milosevic M, Warde P, Menard C, Chung P, Toi A, Ishkanian A, McLean M, Pintilie M, Sykes J, Gospodarowicz M, Catton C, Hill RP, Bristow R (2012) Tumor hypoxia predicts biochemical failure following radiotherapy for clinically localized prostate cancer. Clin Cancer Res 18: 2108-2114.

Mitani T, Yamaji R, Higashimura Y, Harada N, Nakano Y, Inui H (2011) Hypoxia enhances transcriptional activity of androgen receptor through hypoxia-inducible factor-1alpha in a low androgen environment. J Steroid Biochem Mol Biol 123: 58-64.

Park C, Kim Y, Shim M, Lee Y (2012) Hypoxia enhances ligand-occupied androgen receptor activity. Biochem Biophys Res Commun 418: 319-323.

Ragnum HB, Roe K, Holm R, Vlatkovic L, Nesland JM, Aarnes EK, Ree AH, Flatmark K, Seierstad T, Lilleby W, Lyng H (2013) Hypoxia-independent downregulation of hypoxia-inducible factor 1 targets by androgen deprivation therapy in prostate cancer. Int J Radiat Oncol Biol Phys 87: 753-760.

Sartori DA, Chan DW (2014) Biomarkers in prostate cancer: what's new? Curr Opin Oncol 26: 259-264.

Sboner A, Demichelis F, Calza S, Pawitan Y, Setlur SR, Hoshida Y, Perner S, Adami HO, Fall K, Mucci LA, Kantoff PW, Stampfer M, Andersson SO, Varenhorst E, Johansson JE, Gerstein MB, Golub TR, Rubin MA, Andren O (2010) Molecular sampling of prostate cancer: a dilemma for predicting disease progression. BMC Med Genom 3: 8.
Shariat SF, Lotan Y, Saboorian H, Khoddami SM, Roehrborn CG, Slawin KM, Ashfaq R (2004) Survivin expression is associated with features of biologically aggressive prostate carcinoma. Cancer 100: 751-757.

Sobin LH, Gospodarowicz MK, Wittekind C (2009) TNM Classification of Malignant Tumours. 7th ed, John Wiley \& Sons Ltd: West Sussex, UK.

Sonn GA, Margolis DJ, Marks LS (2014) Target detection: magnetic resonance imaging-ultrasound fusion-guided prostate biopsy. Urol Oncol 32: S1078-S1439.

Srigley JR (2006) Key issues in handling and reporting radical prostatectomy specimens. Arch Pathol Lab Med 130: 303-317.

Starmans MH, Krishnapuram B, Steck H, Horlings H, Nuyten DS, van d V, Seigneuric R, Buffa FM, Harris AL, Wouters BG, Lambin P (2008) Robust prognostic value of a knowledge-based proliferation signature across large patient microarray studies spanning different cancer types. Br J Cancer 99: 1884-1890.

Toubaji A, Sutcliffe S, Chaux A, Lecksell K, Hicks J, De Marzo AM, Platz EA, Netto GJ (2012) Immunohistochemical expression of minichromosome maintenance complex protein 2 predicts biochemical recurrence in prostate cancer: a tissue microarray and digital imaging analysis-based study of 428 cases. Hum Pathol 43: 1852-1865.

Toustrup K, Sorensen BS, Nordsmark M, Busk M, Wiuf C, Alsner J, Overgaard J (2011) Development of a hypoxia gene expression classifier with predictive impact for hypoxic modification of radiotherapy in head and neck cancer. Cancer Res 71: 5923-5931.

Turaka A, Buyyounouski MK, Hanlon AL, Horwitz EM, Greenberg RE, Movsas B (2012) Hypoxic prostate/muscle PO2 ratio predicts for outcome in patients with localized prostate cancer: long-term results. Int $J$ Radiat Oncol Biol Phys 82: e433-e439.

Urbanucci A, Sahu B, Seppala J, Larjo A, Latonen LM, Waltering KK, Tammela TL, Vessella RL, Lahdesmaki H, Janne OA, Visakorpi T (2012) Overexpression of androgen receptor enhances the binding of the receptor to the chromatin in prostate cancer. Oncogene 31: 2153-2163.

van der Kwast TH, Amin MB, Billis A, Epstein JI, Griffiths D, Humphrey PA, Montironi R, Wheeler TM, Srigley JR, Egevad L, Delahunt B (2011) International Society of Urological Pathology (ISUP) Consensus Conference on Handling and Staging of Radical Prostatectomy Specimens. Working group 2: T2 substaging and prostate cancer volume. Mod Pathol 24: $16-25$.

van Laarhoven HW, Kaanders JH, Lok J, Peeters WJ, Rijken PF, Wiering B, Ruers TJ, Punt CJ, Heerschap A, van der Kogel AJ (2006) Hypoxia in relation to vasculature and proliferation in liver metastases in patients with colorectal cancer. Int J Radiat Oncol Biol Phys 64: 473-482.

Verhoven B, Yan Y, Ritter M, Khor LY, Hammond E, Jones C, Amin M, Bahary JP, Zeitzer K, Pollack A (2013) Ki-67 is an independent predictor of metastasis and cause-specific mortality for prostate cancer patients treated on Radiation Therapy Oncology Group (RTOG) 94-08. Int $J$ Radiat Oncol Biol Phys 86: 317-323.

Wijffels KI, Marres HA, Peters JP, Rijken PF, van der Kogel AJ, Kaanders JH (2008) Tumour cell proliferation under hypoxic conditions in human head and neck squamous cell carcinomas. Oral Oncol 44: 335-344.

Winter SC, Buffa FM, Silva P, Miller C, Valentine HR, Turley H, Shah KA, Cox GJ, Corbridge RJ, Homer JJ, Musgrove B, Slevin N, Sloan P, Price P, West CM, Harris AL (2007) Relation of a hypoxia metagene derived from head and neck cancer to prognosis of multiple cancers. Cancer Res 67: 3441-3449.

Xia L, Mo P, Huang W, Zhang L, Wang Y, Zhu H, Tian D, Liu J, Chen Z, Zhang Y, Chen Z, Hu H, Fan D, Nie Y, Wu K (2012) The TNF-alpha/ ROS/HIF-1-induced upregulation of FoxMI expression promotes HCC proliferation and resistance to apoptosis. Carcinogenesis 33: 2250-2259.

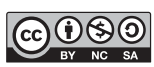
This work is licensed under the Creative Commons Attribution-NonCommercial-Share Alike 3.0 Unported License. To view a copy of this license, visit http://creativecommons. org/licenses/by-nc-sa/3.0/

Supplementary Information accompanies this paper on British Journal of Cancer website (http://www.nature.com/bjc) 